\title{
Organophosphorus pesticides residue in cattle milk from Khartoum state
}

\author{
Sara A. M ${ }^{1}$, Afaf I. A ${ }^{2}$, Sahar M. A ${ }^{1}$, Abubakar A. S ${ }^{1}$, Abdelbagi A. O²., Abdalla A. $\mathbf{M}^{2}$. \\ Corresponding Author: afafizzeldin@yahoo.com \\ ${ }^{1}$ Central Veterinary Research Laboratory \\ ${ }^{2}$ University of Khartoum \\ DOI: $10.31364 / \mathrm{SCIRJ} / \mathrm{v} 7.11 .2019 . P 0119603$ \\ http://dx.doi.org/10.31364/SCIRJ/v7.i1.2019.P0119603
}

\begin{abstract}
The current study was performed to determine the kinds of organophosphorus pesticides residue in milk sample in state of Khartoum-Sudan. 66 samples were collected from breeding farms widespread the state, regarding the areas of high farms concentration. Samples were collected in sterile vacutainers frigid, extracted and analyzed using gas chromatograph GC/FID. Two pesticides belong to organophosphorus group were detected (Dimethoate and Chloropyrifos). $4 \%$ of Khartoum bahri samples contained the two pesticides, while Khartoum and Omdurman recorded $21 \%$ and $5.26 \%$ respectively. $92 \%$ of dimethoate positive samples from Khartoum Bahri area exceeded the maximum residue limits, while Khartoum and Omdurman showed the same percentage $84.2 \%$. All Choloropyrifos positive samples were below the maximum residue limits.
\end{abstract}

Key words: Organophosphorus, Milk, Gas Chromatography, Khartoum state

\section{1-Introduction:}

Pesticides are used worldwide to increase the production but many persistent residues of pesticides cause problem in environmental contamination and human health. Several pesticides contain the noxious substances that persist in environment for a long time so pesticides contamination is a worldwide public health concern and also a main international trade problem. Therefore, from health point of view, the control of pesticides application is a necessity (Latif et al, 2011).

Organophosphorus pesticides usage has a huge advantage in term of providing a high product quality through the protective role of plants against insects. When dairy cows are fed with organophosphorus pesticide polluted forages or drinking water, bovine milk and dairy products might be tainted with pesticide residues (Enid turyahikayo, 2013).

The international quality standards of pesticides application, is followed only by $30 \%$ of the developing countries. The human, animal and environment as well are under huge risk due the magnitude, poorly controlled application, which leads to health threats because of the accumulative effect (Jivraj \& Astha, 2017).

Several studies had been carried out to monitor the concentration of pesticides residues in biological fluids including cow milk, as a marker of pesticides persistence in environment. The substances intended for preventing, destroying, repelling pests are known as pesticides. Several hundred pesticides of different chemical nature are currently used for agricultural purposes all over the world. Because of their widespread use, they are detected in various environmental matrices, such as soil, water and air. Pesticides are divided into many classes, of which the most important are organochlorine and organophosphorous compounds (Kotinagu et al, 2018). 
The presence of pesticides residue in milk and milk products; represents an urgent issue for both the producers and the consumers, this issue needs to be solved to prevent the health risks (deposition of residues in human body fats), which could be raised if it neglected. The heath prospective problems from accumulation of residue in human body are endocrine disrupt, heart problems and cancer. (Neelam et al, 2013).

Information about the kinds and quantities of pesticides in the environment and or food could be obtained by milk tracking. The abstracted information is beneficial in predicting health problems linked to the milk consumption subscription for pesticides intake into human body during the different stages of their lives. That's why the divination of environmental chemicals in common milk types and milk products, gets a tremendous scientific attention and interest (Moustafa, 2017), so this study aimed to investigate the types of organophosphorus pesticides used in agricultural sector in Khartoum state, and to screen the situation of those pesticides residue in milk samples, from prospect of their presence in milk exceed the maximum residue limits which determined by the international authorities or not.

\section{2- Materials and method:}

2-1. Study Area: The Khartoum state with its three big towns (Khartoum, Khartoum Bahri and Omdurman), was the targeted area of this surveillance. Probability sampling method was followed, to insure optimum coverage of all areas.

2-2. Chemicals: All chemical used for this analysis were GC grade.

2-3. Standards: pesticides standards were obtained from department of crops protection, college of agriculture-University of Khartoum

2-4. Sample Collection: A total of 66 milk samples were collected in sterilized $500 \mathrm{ml}$ containers, and immediately transferred to refrigerator to avoid any other environmental contaminations.

\section{2-5. GC analysis condition:}

\begin{tabular}{|l||l||}
\hline Detector & FID \\
\hline \hline Column & DB-5, Film thickness 0.25um, Inner diameter 0.25mm, Column length 30m. \\
\hline \hline Carrier gas & N2/Air \\
\hline \hline Injection mode & Spilt \\
\hline
\end{tabular}

\section{2-6. Sample preparation:}

2-6-1. Extraction: A $(500 \mathrm{~mL})$ of milk sample was transferred into a reparatory funnel. (50 ml) of Petroleum ether was added and shaken well. Another portion of petroleum ether $(200 \mathrm{~mL})$ was added and Sodium Chloride ( $\mathrm{NaCl})$ was also added to boost separation. The sample was rotated and allowed to settle for overnight. The lower layer discarded. Petroleum ether (200 mL) was added to the top layer, and $\mathrm{NaCl}(2 \mathrm{~g})$ was also added to boost separation. The lower layer was drained out. This process was repeated three times and the top layer was collected in a $250 \mathrm{~mL}$ volumetric flask. The petroleum ether was concentrated to $1 \mathrm{ml}$ using rotary evaporator (AOAC, 1995)

2-6-2. Partitioning: Partitioning of the extracted milk samples was carried out according to (Leon et al. 1990). 
To the petroleum ether concentrated sample, a $(10 \mathrm{~mL})$ Acetonitril was added and again concentrated to $5 \mathrm{ml}$. $(3 \mathrm{~mL})$ petroleum ether was added and the mixture was mixed well and allocated in the freezer for two hours, the petroleum ether was dismissed to remain with acetonitrile to remove the fats. The extract was further concentrated to $3 \mathrm{~mL}$. (Enid turyahikayo, 2013).

2-6-3. Cleanup: The extracts, about $(5 \mathrm{ml})$ were transferred to silica gel cartridge (pre-washed with petroleum either and acetonitrile (75: 25 by volume; $10 \mathrm{ml})$ ) and eluted with petroleum either and acetonitrile $(75: 25$ by volume; $25 \mathrm{ml})$ at rate $510 \mathrm{ml} / \mathrm{min}$. The eluant was collected and evaporated to dryness with a rotary vacuum evaporator at $60^{\circ} \mathrm{C}$. The residue was dissolved in $3 \mathrm{ml}$ acetonitrile, and filtered through a membrane filter $(0.45 \mu \mathrm{m})$ and analyzed by gas chromatography.

\section{Statistical analysis:}

The data were analyzed by using Statistics-10 program designed for Windows. Differences between obtained values were carried out by analysis of variance (ANOVA) the LCD test was used for determining the significance level of at least $p<0.05$.

\section{4- Results and discussion:}

In agricultural sector; different types of materials from synthetic chemical origin are used. These substances are known as pesticides; this term (Pesticides) include herbicides, rodenticides, fungicides and insecticides. Applying pesticides throughout the different stages of agricultural processes, and even after harvesting; is companioned with the ability of these substances to convey from lower plants and animals to the higher plants and animals among the food chain and gather in higher organisms. The direct application of pesticides; such as spray animals habitations to control the pests, also leads to gathering of the residues in animal's products such as milk, egg and meat. That's why the dietary rout represents a pivotal way of chronic exposure to pesticides (Shazia and Karam, 2017).

Generally, all samples were positive. Two types of organophosphorus pesticides were detected. Dimethoate and Chloropyrifos.92.3\% of Khartoum Bahri samples were Dimethoate positive, $34.78 \%$ out of them exceeded the maximum residue limits of dimethoate in milk samples, which determined by (Codex, 2006); (0.05 ppm). In regard to Khartoum city (84.2\%) of samples were dimethoate positive, $25 \%$ exceeded the (MRL). Omdurman recorded $84.2 \%$ dimethoate positive, but no one was beyond the (MRL). The Dimethoate level in milk samples collected from Omdurman city, was remarkably low compared to those collected from Bahri and Khartoum, the difference was significant at $(\mathrm{p}<0.05)$.

Dimethoate is an organophosphorous insecticide that is highly water soluble. At room temperature, it is stable in aqueous solutions of pH 2-7; however, it is unstable under alkaline conditions. It has a low affinity for soils and a moderate affinity for organic matter. It is susceptible to hydrolysis under acidic conditions, is moderately stable to microbial degradation and is non-volatile as reflected by its low vapor pressure (US EPA 2008), depending on these physical and chemical properties, the presence of this pesticides in almost all analyzed samples even at levels exceeds the (MRL), is justified. Its low affinity to soil plus the type of soil on which those farms are build, may increase the incidence of pesticide leakage into the ground water, and the later one could be used for drinking by both animals and human, for aggregation of crops around farms from which animals feed is coming. Plus the $\mathrm{PH}$ of cow milk which range between (6.5-6.7) provides stability condition for the pesticide.

In another study by (Rafael et al, 2011), they reported 25\% out of 30 milk samples collected from different dairy farms from different areas in Spain, were dimethoate positive. On another hand, chloropyrifos was detected in 4\%, 26.3\% and 15.78\% of Bahri, Khartoum and Omdurman samples respectively. All levels were less than maximum residue level of chloropyrifos recommended by Codex $(0.2$ ppm). No significant different level of chloropyrifos between all groups was observed. Shahzadi and her co workers reported that, (chlorpyrifos) in buffalo, sheep, cow, goat and camel milk samples was detected. Those samples were collected from different 
locations of Lahore and identified by High performance thin layer chromatography (HPTLC), Ultraviolet- visible (UV) and GC-MS were used to analyze the residues contamination level. Another study by Neelam and his co workers carried out in Faisalabad, cattle milk was collected from different localities of Faisalabad, Pakistan and extracted using solid phase micro-extraction technique, to detect pesticides residue by using HPLC. The results of this study showed that overall $40 \%$ samples recorded pesticides contamination. The mean level of chloropyrifos was $0.072 \mu \mathrm{g} / \mathrm{mL}$ (Shahazdi et al, 2013).

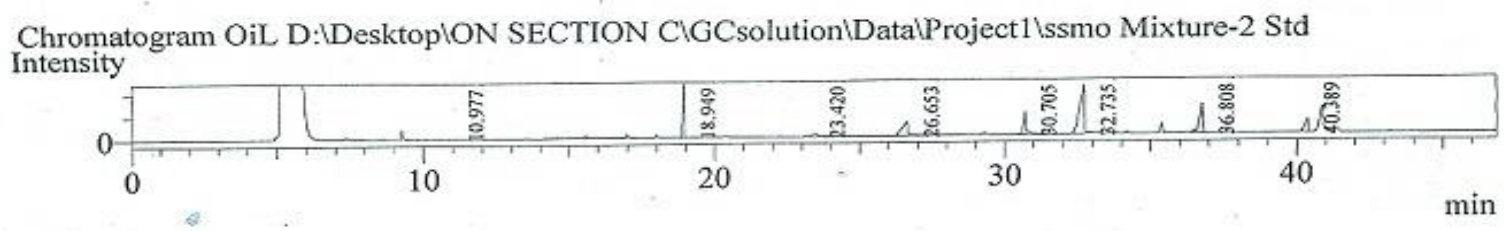

\begin{tabular}{|r|r|r|r|r|l|l|l|}
\hline \multicolumn{10}{|c|}{ Peak\# } & Ret.Time & \multicolumn{1}{|c|}{ Area } & \multicolumn{1}{|c|}{ Height } & \multicolumn{1}{c|}{ Conc. } & Units & Mark & Name \\
\hline 1 & 10.977 & 51 & 44 & 0.000 & & & \\
\hline 2 & 18.949 & 1709523 & 469147 & 283.507 & $\mathrm{mg} / \mathrm{ml}$ & Diazinone \\
\hline 3 & 23.420 & 317541 & 19205 & 1010.108 & $\mathrm{mg} / \mathrm{ml}$ & Omethoate+DDA \\
\hline 4 & 26.653 & 1762631 & 122397 & 2475.652 & $\mathrm{mg} / \mathrm{ml}$ & Dimethoate \\
\hline 5 & 30.705 & 1359276 & 191846 & 141.671 & $\mathrm{mg} / \mathrm{ml}$ & Heptachlor \\
\hline 6 & 32.735 & 4535127 & 425675 & 418.383 & $\mathrm{mg} / \mathrm{ml}$ & Chlorpyrifos \\
\hline 7 & 36.808 & 2095083 & 254026 & 259.208 & $\mathrm{mg} / \mathrm{ml}$ & & Endosulfan alpha \\
\hline 8 & 40.389 & 828486 & 108621 & 126.393 & $\mathrm{mg} / \mathrm{ml}$ & & Endosulfan Beta \\
\hline Total & & 12607718 & 1590961 & & & & \\
\hline
\end{tabular}

Figure (1): Elution pattern of standards mixture.

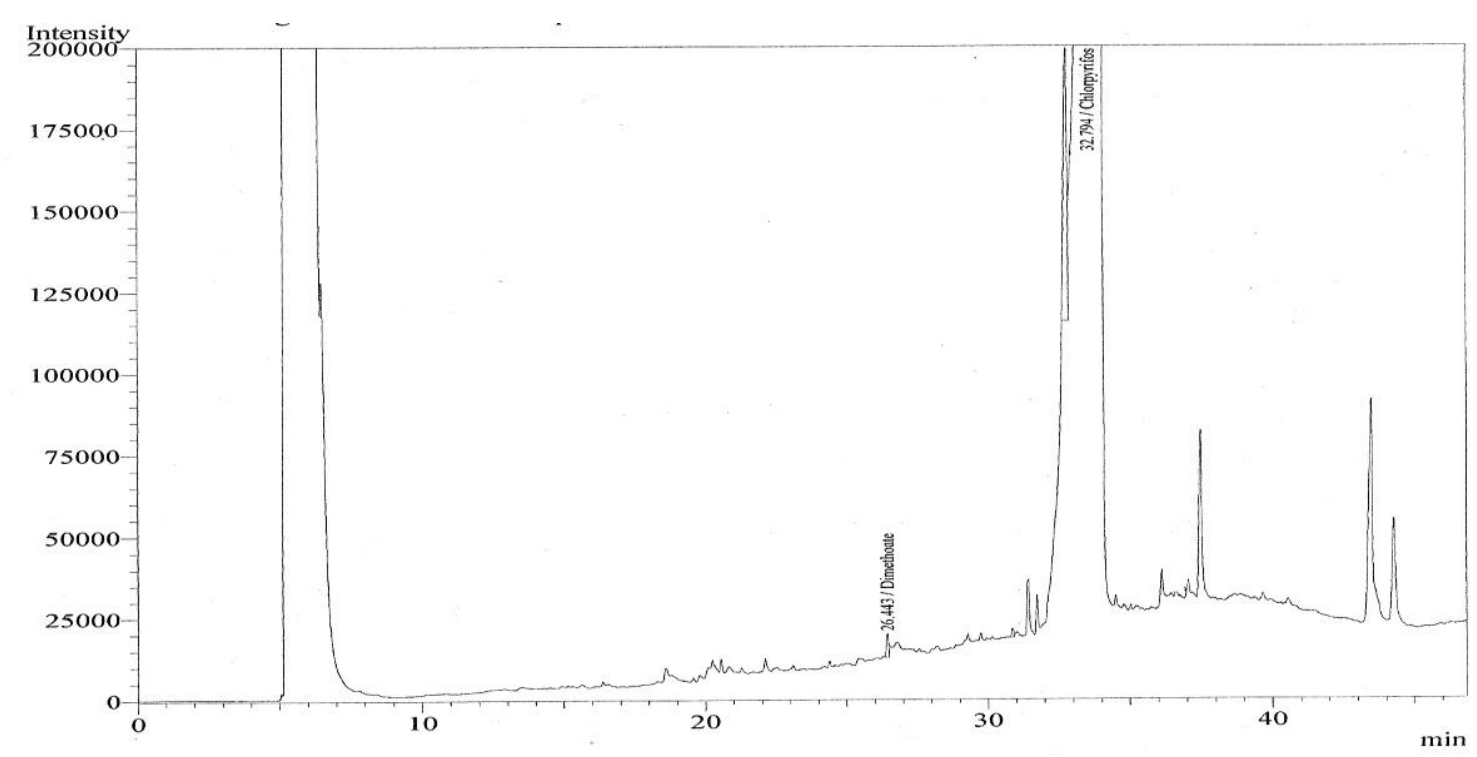

Figure (2): Elution pattern of organophosphorus pesticide residue (Dimethoate + chlorpyrifos) in milk samples from Omdurman city. 
Table (1):

\begin{tabular}{|l|c|c|c|}
\hline \multicolumn{1}{|c|}{ Pesticide Name } & Molecular Formula & Molecular Weight & Codex (MRL) \\
\hline Dimethoate & $\underline{\mathrm{C}}_{2} \underline{\mathrm{H}}_{12} \underline{\mathrm{NO}}_{3} \underline{\mathrm{PS}_{2}} \underline{2}_{2}$ & $229.249 \mathrm{~g} / \mathrm{mol}$ & $0.05 \mathrm{ppm}$ \\
\hline \hline Chlorpyrifos & $\underline{\mathrm{C}}_{2} \underline{\mathrm{H}}_{11} \underline{\mathrm{Cl}}_{3} \underline{\mathrm{NO}}_{2} \underline{\mathrm{PS}}$ & $350.575 \mathrm{~g} / \mathrm{mol}$ & $0.2 \mathrm{ppm}$ \\
\hline
\end{tabular}

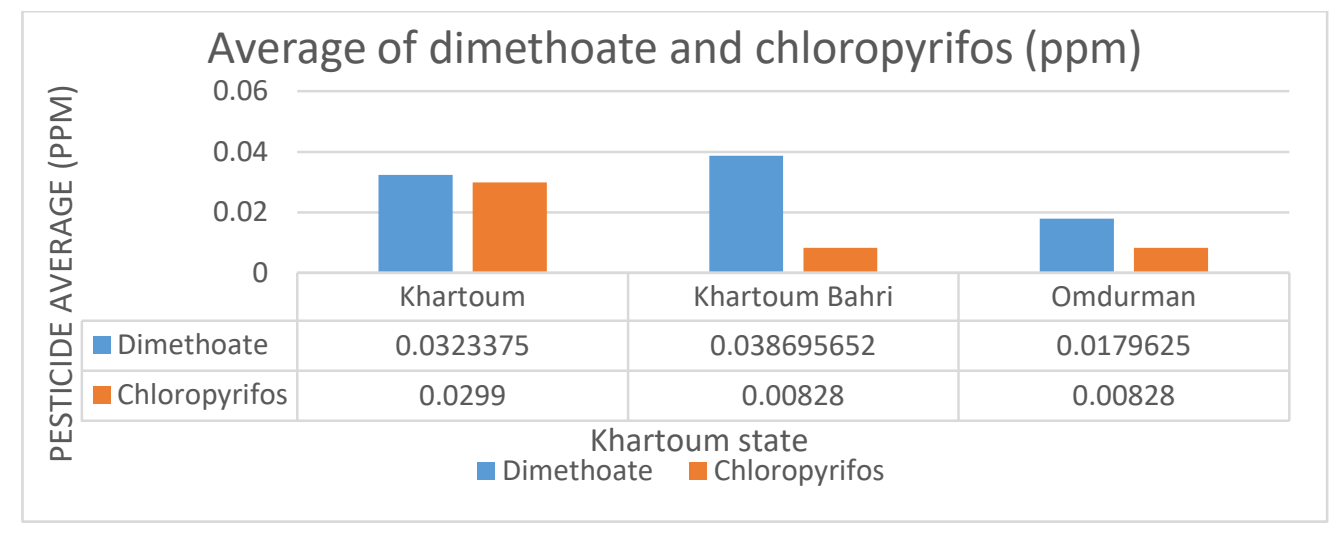

Figure (3): Average of detected pesticides in milk samples

\section{References:}

1- A.O.A.C. (1995). Official methods of the association Official Analytical Chemists. Washington. D.C

2- Codex. (2006). Maximum residue limits for pesticide residues in food.

3- Jivraj Makadiyal and Astha Pandey1. (2018). Detection of pesticides in buffalo milk collected from different areas of gandhinagar and Ahmedabad by thin layer chromatography. World journal of pharmacy and pharmaceutical sciences SJIF, 7 (3): 467-478.

4- Kotinagu Korrapati, Karthik Kotha and Krishnaiah Nelapati. (2018). Determination of organophosphorus pesticide residues in milk samples along Musi river belt, Hyderabad, India, The Pharma Innovation Journal; 7(4): 242-246

5- Latif Y, Sherazi STH, Bhanger MI. (2011). Monitoring of pesticide residues in commonly used Fruits in Hyderabad Region, Pakistan. American Journal of Analytical Chemistry.2, 46-52.

6- Moustafa M.S. Abbassy. (2017). Pesticide Residues in Buffalo and Human Breast Milk of Vegetables and Fruits Farming Community at Northern of Delta in Egypt. Journal of Environmental \& Analytical Toxicology. Abbassy, J Environ Anal Toxicol 2017, 7:2.

7- Neelam Shahzadi, Muhammad Imran, Mohammad Sarwar, Abu Saeed Hashmi, Muhammad Wasim. (2013). Identification of pesticides residues in different samples of milk. Journal of Agroalimentary Processes and Technologies, 19(2), 167-172.

8- Rafael F, Vanerli B Ana P. P Karen D, and Ronaldo T. (2011). Organophosphorous and carbamates residues in milk and feedstuff supplied to dairy cattle. J of Pesquisa. Veterinaria. Brasileira, 31(7): 598-602.

9- Shahzadi N, Imran M, Sarwar, M., Hashmi A. S, and Wasim M. (2013). Identification of pesticides residues in different samples of milk J. Agroalimentary Processes and Technology., 19(2): 167. 
10- Shazia Akhtar and Karam. (2017). Pesticides Residue in Milk and Milk Products: Mini Review. Pak. J. Anal. Environ. Chem. 18(1): $37-45$.

11- US environmental protection agency (EPA). (2008). Labeling Amendment; revision according to Dimethoate.

12- Enid Turyahikayo. (2013). Assessing the levels of pesticide residues in livestock products and water around lake MBURO National Park, south western UGANDA. A dissertation submitted to Makerere University, College of agriculture and environmental sciences. 